\title{
Examiners and assessors
}

The General Medical Council has asked the College for further nominations to the list of Examiners and Assessors, in connection with the Council's health procedures. Those interested are invited to let the College Secretary know that they wish to be considered for nomination. It may be helpful to give some further information on what the work involves.

Professor Kessel has described some aspects of the health procedures (Psychiatric Bulletin, $1996,20,577-579)$. As he states, where a prima facie case of possible impaired fitness to practise has been established, doctors are invited to be examined by at least two medical examiners, usually psychiatrists, with specialists in other appropriate fields if necessary.

Examiners are asked to provide a comprehensive psychiatric report, taking into account examination of the doctor, information from other sources - for example, relatives, friends and colleagues. They are asked to indicate diagnosis and possible treatment options and to answer specific questions concerning fitness to practise and possible restrictions on practice. Although many referred doctors have problems of substance misuse, the GMC requires a general comprehensive report, and few current examiners are specialists in this area - nor need they be.

Examiners are contacted in advance by GMC staff and invited to examine the specific doctor. This gives them the opportunity of indicating if they know the doctor, or if it is not convenient for some reason to arrange an examination at that time. It is helpful if the report, for which a fee is payable, can be sent to the GMC within 5 weeks and so annual leave etc, may make it difficult. Whenever possible, examiners are selected to reflect geographical considerations - it is usually kinder not to expect referred doctors to travel great distances for examination. Examiners, however, must be independent-not having previous acquaintance with the doctor, either personally or professionally. For these reasons, and also to avoid a small number of examiners constantly being called upon, the General Medical Council wishes to increase the number of examiners.

Psychiatrists are also needed to act as Assessors for the Health Committee. Assessors sit with the Health Committee at its formal hearings in order to advise members of the Committee on the doctor's health and fitness to practise, as revealed in the medical reports and other evidence before them. The assessors are chosen with regard to the nature of the physical or mental condition alleged to impair the doctor's fitness to practise and the doctor's own field of practice. In the first category, there is invariably a psychiatrist.

The Royal College is looking for interested psychiatrists whom it is suggested should have held a consultant or equivalent post for at least 5 years in any of the specialities of psychiatry. I have acted as Examiner and Assessor for the last 12 years and can testify to the importance and interest of the work. I would be happy to discuss any aspects with colleagues who might be interested and can be contacted either through the Royal College or at the address below.

S. A. Mann, The Landermere Centre, Clacton Hospital, Clacton on Sea, Essex CO15 $1 \mathrm{LH}$ (Tel: 01255253601 ) 